\title{
Planification de projets de développement touristiques régionaux : par qui? pour qui?
}

\author{
Normand Bourgault, professeur-chercheur \\ Université du Québec en Outaouais
}

\section{INTRODUCTION}

\section{La Commission régionale sur les ressources naturelles et le territoire public de l'Outaouais (CRRNTO) a invité notre groupe de recherche à établir un portrait d'ensemble des projets de développement récréotouristiques.}

L'Outaouais, tout comme la plupart des régions du Québec, possède un territoire public vaste et riche qui a beaucoup à offrir en termes de récréotourisme en milieu naturel. Ce ne sont pas les projets qui manquent. D'ailleurs, c'est plutôt ce foisonnement d'idées et de projets qui, paradoxalement, pose problème. Quelle direction emprunter, que prioriser, comment dynamiser et supporter? La Commission régionale sur les ressources naturelles et le

\section{LE CADRE D'ANALYSE}

Au départ, notre projet de recherche visait essentiellement à réaliser une synthèse des multiples études de marché réalisées au cours de la dernière décennie pour permettre aux décideurs d'évaluer les voies qui s'ouvraient à eux. Rapidement, nous avons conclu à la nécessité d'enrichir notre problématique de recherche et de répondre à la question: est-ce que le modèle théorique de faisabilité de projet peut enrichir la pratique de la planification de projets récréotouristiques en milieu naturel?

\subsection{La Méthodologie}

Notre méthodologie d'examen des multiples études de faisabilité de projet rédigées au cours de la dernière décennie est celle de l'analyse documentaire. Nous avons considéré la documentation émanant de la CRÉO, des quatre municipalités régionales de comtés (MRC), des organismes voués au développement comme les centres locaux de territoire public de l'Outaouais (CRRNTO) a invité notre groupe de recherche à établir un portrait d'ensemble des projets de développement récréotouristiques ${ }^{1}$ qui ont été élaborés au cours des années et impliquant le territoire couvert par la Conférence régionale des élus de l'Outaouais (CRÉO). La finalité de cette recherche n'était pas de choisir entre les projets, mais plutôt d'aider à la prise de décision, à savoir identifier les conditions qui assurent une bonne fin aux projets récréotouristiques en milieu naturel.

Les résultats de cette recherche seraient limités et anecdotiques s'ils ne s'appliquaient qu'à la seule région étudiée, l'Outaouais. L'examen sommaire de la documentation produite dans les autres régions montre qu'elles utilisent les mêmes méthodes.

développement (CLD), les sociétés d'aide au développement des collectivités (SADC) et de Tourisme Outaouais. Nous avons intégré à nos recherches les documents publics produits par les organismes faisant la promotion d'activités particulières comme les fédérations sportives, les zones d'exploitation contrôlées (ZEC) ou encore les entreprises de récréotourisme comme les pourvoiries. Nous avons aussi dépouillé les études traitant de projets récréotouristiques localisés comme les projets de parcs ou de sites de villégiature.

Nous avons donc repéré et acquis toutes les études de planification et de développement rendues publiques depuis les années 2000 et impliquant des activités récréotouristiques sises sur le territoire de l'Outaouais. Notre recension d'écrits nous a ramené plus de 55 études totalisant plus de 6000 pages. À cause des pratiques commerciales et du secret entourant la réalisation d'études privées, nous n'avons pu accéder à toutes les études. 


\subsection{L'approche gestion de projet comme cadre théorique}

Comment analyser des centaines de projets sans un cadre conceptuel souple et adaptable à diverses situations de gestion? L'approche de gestion de projet s'est imposée d'elle-même. Pour poser un diagnostic, nous avons comparé le contenu théorique d'une faisabilité complète de projets avec le contenu des documents de planification analysés de manière à faire ressortir les similarités et les écarts. Rappelons qu'une étude complète de faisabilité de projet contient quatre études développées en concomitance.

- L'étude de faisabilité technique décrit comment le projet est réalisable.

- L'étude de faisabilité de ressources humaines se penche sur la disponibilité et la compétence du personnel nécessaire à la bonne marche du projet.

- L'étude de faisabilité financière présente les hypothèses de montage financier.

- L'étude de faisabilité de marché, généralement appelée étude de marché, dresse un tableau du marché potentiel pour le produit ou le service et en évalue la rentabilité. Elle vise essentiellement à comprendre les attentes des clients, usagers et parties prenantes au projet.
L'étude de faisabilité de projet se présente essentiellement comme un état prévisionnel des résultats (figure 1) réalisé à partir des données fournies par les autres études de faisabilité, technique, financière, de ressources humaines et de marché.

En contexte de projet social, l'utilité pour la collectivité et le nombre d'usagers constituent les critères de décision de mise en œuvre. En contexte de projet à caractère économique, la rentabilité du projet détermine la décision d'aller de l'avant.

L'analyse «fine » des profils des consommateurs ou usagers détermine les produits et services à offrir. Les études de faisabilité technique, financière et de ressources humaines auront à spécifier comment la prestation de services sera réalisée. Par exemple, le type d'embarcations à offrir à une clientèle lourdement handicapée diffère totalement de celui à mettre à la disposition d'athlètes internationaux. C'est dire que les solutions techniques, humaines et financières des études de faisabilité ne doivent pas être développées indépendamment des clientèles. Elles sont totalement interdépendantes.

Figure 1

\begin{tabular}{|c|c|c|}
\hline Informations & $\begin{array}{c}\text { Données } \\
\text { quantitatives }\end{array}$ & État prévisionnel des résultats \\
\hline $\begin{array}{l}\text { Revenus potentiels annuels (ventes ou } \\
\text { fréquentation) } \\
\text { Description des segments visés et de } \\
\text { leurs attentes } \\
\text { Dénombrement des acheteurs } \\
\text { potentiels } \rightarrow \\
\text { Montant par transaction } \rightarrow \\
\text { Nombre de transactions annuelles } \rightarrow\end{array}$ & $\begin{array}{r}\begin{array}{r}\text { Nombre } \\
\text { d'acheteurs }\end{array} \\
\mathrm{x} \text { Montant par } \\
\text { transaction } \\
\mathrm{x} \text { Nombre de } \\
=\frac{\text { transactions }}{\rightarrow}\end{array}$ & \multirow{5}{*}{$\begin{array}{l}\text { Revenus potentiels annuels } \\
\frac{\text { - Coût des marchandises }}{\text { (services) vendues }}\end{array}$} \\
\hline $\begin{array}{l}\text { Coût des marchandises vendues ou des } \\
\text { services rendus } \\
\text { Coûts variables des produits et } \\
\text { services offerts } \\
\quad \text { décrits dans les études de faisabilité } \\
\text { en fonction des } \\
\quad \text { attentes de la clientèle visée }\end{array}$ & 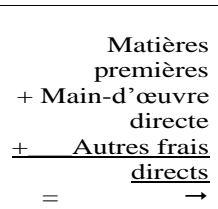 & \\
\hline & & \\
\hline $\begin{array}{l}\text { Frais d'exploitation (coûts fixes) } \\
\text { Frais fixes d'exploitation décrits } \\
\text { dans les études de } \\
\text { faisabilité en fonction des attentes } \\
\text { de la clientèle } \\
\quad \text { visée }\end{array}$ & $\begin{array}{r}\text { Frais de vente } \\
+ \text { Frais } \\
\text { d'administration } \\
+\quad \text { Frais } \\
\text { financiers } \\
+ \\
\text { Loyer } \\
\pm \\
\quad \text { Énergie } \\
\rightarrow\end{array}$ & \\
\hline & & \\
\hline
\end{tabular}


Lors de l'examen des projets récréotouristiques, nous avons accordé une attention particulière aux enjeux concernant les usagers. Pour y arriver, nous avons comparé le contenu des projets au contenu théorique d'une étude de planification stratégique (figure 2), étude qui se trouve au cœur de l'étude de faisabilité de marché.

\section{Figure 2}

ANALYSE EXTERNE
Macro-environnement (PESTE)
- Politique
- Économique
- Socioculturel
- Technologique
- Écologique

Micro-environnement (profil concurrentiel)

- Clientèle

- Segments

- Motivations, attitudes, styles de vie

- Besoins insatisfaits

- Fournisseurs - distributeurs

- Systèmes de distribution, tendances

- Grandeur, croissance

- Barrières à l'entrée

- Compétiteurs

- Identité, groupes stratégiques, maillages \& réseaux

- Performance, image

- Mission, objectifs, stratégies, culture

- Structure de coût

- Forces, faiblesses et contraintes

- Produits-marchés

- Grandeur, tendances et croissance

- Rentabilité \& structure de coût

- Barrières à l'entrée

- Facteurs clés de succès ou d'échecs

Opportunités, menaces et questions stratégiques

\begin{abstract}
ANALYSE INTERNE
Analyse de performance

- Ventes \& rentabilité

- Analyse de la valeur

- Satisfaction des clients

- Qualité des produits-services

- Valeur des marques et de l'image corporative

- Coûts comparatifs (au secteur)

- Nouveaux produits-services

- Portefeuille de produits et cycle de vie

- Capacités et performances du personnel Analyse par fonctions d'entreprise : (production, marketing, management, ressources humaines, comptabilité, finance)
\end{abstract}

Déterminants des options stratégiques

- Mission passée et présente

- Stratégies passées et présentes

- Problèmes stratégiques

- Capacités \& contraintes de la firme

- Ressources \& contraintes financières

\begin{tabular}{|c|c|}
\hline ites, menaces & $\begin{array}{c}\text { Forces, raiplesses, proplemes, contraintes et } \\
\text { questions stratégiques }\end{array}$ \\
\hline $\begin{array}{c}\downarrow \\
\text { IDENTIFICATION ET CHOI } \\
\text { - } \quad \text { Révision de la mission } \\
\text { - } \quad \text { Identification des alternatives stratégiques } \\
\text { - Stratégies d'investissement dans l } \\
\text { - Stratégies pour les différentes fon } \\
\text { - Avantages, inconvénients, synergi } \\
\text { - Choix d'une stratégie } \\
\text { - Implantation : plan marketing } \\
\text { - Contrôle et évaluation des stratégies }\end{array}$ & $\begin{array}{l}\text { DE STRATÉGIES } \\
\text { roduits - marchés } \\
\text { ns de l'entreprise } \\
\text { des alternatives }\end{array}$ \\
\hline
\end{tabular}


Nous avons ensuite procédé à une analyse de contenu. Nous avons recherché dans cette abondante documentation les intentions des institutions concernant le développement récréotouristique de leur territoire. Nous avons extrait de ces documents plus de 250 pages d'informations et de références sur ces projets de développement. Globalement, notre analyse de contenu s'est déroulée en deux temps :

1. Une analyse descendante: de l'ensemble de la région aux communautés locales. L'analyse descendante a consisté à examiner les documents de planifications produits par des organismes couvrant l'ensemble de la région, puis se situant sur des subdivisions territoriales, pour en analyser les ramifications.

2. Une analyse ascendante: des activités et projets locaux aux activités régionales. Nous

\section{L'ANALYSE DU CONTENU DES PROJETS}

Nous avons construit notre analyse autour de deux périodes de planification, 2000-2007 et 2007-2012. L'analyse du contenu des documents décrivant la faisabilité de divers projets de développement récréotouristiques en milieu naturel a montré l'évolution des préoccupations des intervenants locaux et régionaux.

\subsection{0-2007 : la période des inventaires}

Ces analyses techniques ont permis aux planificateurs de s'approprier les connaissances nécessaires à la compréhension des lieux en fonction des multiples dimensions physico-chimiques, géologiques, historiques, culturelles, réglementaires, etc.

Le premier constat qui se dégage des projets produits de 2000 à 2007 est que ces plans constituent, à la base, des inventaires de possibilités et de potentialités. Certains projets sont effleurés, d'autres sont abordés en profondeur. Sauf de rares exceptions, ces études prennent la forme d'analyses de faisabilité techniques sans autres études de faisabilité.

Ces analyses techniques ont permis aux planificateurs de s'approprier les connaissances nécessaires à la compréhension des lieux en sommes partis des activités localisées sur des territoires restreints comme les projets de développement de sites de pratiques de sports d'eau vive ou de randonnées pédestres pour remonter à un niveau régional afin d'en évaluer les nécessaires synergies.

Notre analyse documentaire a permis de dégager, a posteriori puisqu'ils émergeaient des rapports passés, des enjeux et des orientations. Nous les avons formulés. Afin d'assurer la validité externe de nos constats, nous les avons soumis à l'expertise régionale lors d'une tournée de consultation. Dans chaque MRC, nous avons rencontré divers intervenants liés au récréotourisme en milieu naturel. Ces commentaires ont confirmé les constats de notre analyse descendante et de notre analyse ascendante.

fonction des multiples dimensions physicochimiques, géologiques, historiques, culturelles, réglementaires, etc. Il appert que l'on ne pouvait faire l'économie de ces analyses et qu'il était fondamental de les mener. Elles posaient les jalons des développements futurs.

Les projets évoqués se concentrent sur les permis à obtenir et à donner pour permettre le développement récréotouristique comme l'établissement de sites de villégiature, l'installation de rampes de mise à l'eau et d'abris sommaires, etc. Ils évoquent aussi ce qui pourrait être fait pour développer ou améliorer les sites, mais sans référence à des clientèles particulières ayant des caractéristiques sociodémographiques ou psychographiques définies.

Les aménagements proposés portent essentiellement sur les moyens d'accès (chemins, quais, débarcadères, rampes de mise à l'eau) et de circulation sécuritaires (sentiers, balises, rampes, escaliers). À l'occasion, des points d'eau potable et des équipements sanitaires sont prévus, mais ce n'est pas systématique.

Il se dégage de ces analyses techniques l'idée que ces projets sont conçus pour la desserte d'un nombre très restreint d'usagers en grande forme physique et sans enfants. Dans certains cas, toutefois, les services de support comme 
l'hébergement, la restauration, les équipements sanitaires et les marinas sont signalés. Le cas échéant, ils sont évalués.

Dans le contexte de ces projets, trois études de faisabilité ne sont pas prises en compte alors qu'elles sont des conditions fondamentales à leur réalisation. Ces trois études, minimalement, répondent aux questions suivantes :

Faisabilité des ressources humaines

- Qui peut faire le projet? Connaissances, compétences et expériences.

- Qui veut faire le projet? Individu, collectivité, groupes d'intérêt. But lucratif ou non lucratif. Intérêt, motivation et implication.

- Qui doit faire le projet? Source de motivation intrinsèque (leadership) ou extrinsèque (imposé d'autorité)? Intérêts individuels ou collectifs?

- Qui dispose des moyens de faire le projet? Individus ou groupes d'individus?

Faisabilité financière

- Quelles sont les sommes en jeu?

- Qui finance?

- Encaisse-t-on? Utilisation gratuite, autofinancement ou exigences de rentabilité.

Faisabilité de marché

- Qui seraient les clients? Caractéristiques, attentes par rapport aux services à recevoir et au niveau de qualité des services. Niveaux de prix considérés.

- Pourquoi seraient-ils clients? Avantage concurrentiel du projet comme réponse aux besoins et désirs des clients.

- Comment les recrute-t-on?

- Quelle est la désirabilité sociale de ce projet pour les groupes d'intérêt? Qui sont ces groupes d'intérêt?

L'identification des attentes et désirs des usagers est généralement absente des plans d'aménagement. Les individus ou groupes entrepreneuriaux de mise en œuvre des projets le sont aussi. De même, les sources de financement sont rarement évoquées.

\subsection{7-2012 : la période de l'intégration des faisabilités techniques}

La problématique du développement récréotouristique de plein air apparaît dans un cadre plus large, dans la planification stratégique 2007-
2012. Elle s'est contextualisée et enrichie et les thématiques se sont précisées. Ainsi, les plans de développement intégrés (PDI) décrivent des projets ayant davantage de liens entre eux qu'auparavant. Par exemple, les projets impliquant des rivières prennent ces dernières comme concept intégrateur des sous-projets possibles le long de ses rives. D'autres sont décrits par activités à développer et des lieux à aménager pour y parvenir.

\subsubsection{Des clients conjugués au passé}

Certaines études présentent des activités possibles en région accompagnées de statistiques générales de fréquentation québécoises ou canadiennes. L'offre locale et régionale est comparée à ces données nationales pour apprécier la pertinence des projets.

Dans les études de cette période, le client y est traité de façon incidente. Les besoins du client sont « supposés » à partir de ce qu'il consomme et de ce qui lui est proposé plutôt que décrits par ce qu'il veut acheter. Les besoins des clientèles (demande), tels que présentés, ne permettent pas de préciser le panier de produits et services à développer dans chaque projet pour les satisfaire. Décrire les attentes des clients en fonction des ventes passées limite l'innovation.

\subsubsection{L'arrivée du client, dans certains cas}

Encore durant cette période, la plupart des documents de planification ressemblent à des «catalogues » de projets possibles, sans spécification du « pour qui » ni du « par qui », ils devraient être mis en œuvre. Le client avec ses goûts et ses attentes apparaît comme acteur clé du récréotourisme à partir de 2010. On y présente le nouveau consommateur de récréotourisme et on élabore des offres basées sur des expériences touristiques distinctives ainsi que sur les moyens de communication à mettre en œuvre pour l'amener à les vivre.

\section{Le client avec ses goûts et ses attentes apparaît comme acteur clé du récréotourisme à partir de 2010.}




\subsubsection{Le client ou le citoyen}

Le souci d'ajuster l'offre des services à la population locale à celle des autres localités devient couramment évoqué, ce qui n'est pas le cas des documents de la période 2000-2007. Les projets sont décrits à la fois en termes de bénéfices pour les citoyens et d'attraits touristiques à vocation commerciale.

\subsubsection{Des partenariats sans projet et des projets sans partenariat}

L'idée d'établir des collaborations avec d'autres organismes locaux ou régionaux ressort de la plupart des planifications, en particulier celles des MRC. Elle est très couramment évoquée, mais sans référence à des projets précis. Par exemple, alors que deux municipalités voisines ont chacune un projet de piste cyclable, aucune ne mentionne la nécessité de s'entendre sur le tracé alors que les deux mentionnent la nécessité de mieux collaborer.

À ce sujet, un tableau que nous avons utilisé lors de la tournée régionale de validation de nos constats a surpris la quasi-totalité des participants. Il montre que les MRC ont sensiblement les mêmes projets de développement, aménagement de pistes cyclables, de sentiers pédestres, de parcs, etc. Les commentaires des participants ont mis en lumière la méconnaissance des projets des territoires immédiatement voisins.

\subsubsection{Des résultats généraux, difficilement mesurables}

Les planifications de cette période innovent par la présence d'objectifs et de plans d'action. Ils décrivent des résultats spécifiques. À l'analyse, les résultats décrits dans les objectifs sont difficilement mesurables. Des verbes comme favoriser, soutenir, impliquer, valoriser peuvent décrire des résultats d'objectifs généraux ou sociaux. Comme il est difficile de mesurer le soutien ou l'implication, ces verbes sont inadéquats pour décrire des objectifs spécifiques ou terminaux.
2.2.6 Le souci de l'environnement et de la pérennité

À la lecture des plans de développement, au défilement des centaines de projets pensés, projetés, étudiés, dessinés, il apparaît en filigrane un triple questionnement, un non-dit qui teinte toutes les démarches régionales de planification qu'il nous a été donné de consulter :

- Comment prélever sans piller?

- Comment utiliser sans détruire?

- Comment tirer profit sans brader?

Ce souci de «préserver - développer» est systématiquement pris en compte. La composante «préserver» domine largement. Il en ressort clairement que la tension du «préserver développer » n'est pas résolue.

\subsubsection{Des études incomplètes}

Notre revue des études existantes pour en extraire les données relatives au récréotourisme de plein air nous amène à conclure que ce sont essentiellement des études de faisabilité techniques.

- Elles décrivent des projets possibles;

- Elles présentent plusieurs centaines de possibilités;

- Elles expliquent l'intérêt des sites ou des activités pour des raisons sociales, historiques, environnementales ou autres;

- Elles spécifient les conditions légales et techniques d'accès au site ou à l'activité.

Nous n'avons pas trouvé :

- D'études de faisabilité de ressources humaines;

- D'études de faisabilité financière;

- De planification stratégique du marketing

- D'études de faisabilité de marché.

Force est de constater que les documents de planifications que nous avons consultés, plus de 50, ne contiennent pas les données nécessaires pour évaluer le potentiel économique de ces projets ou l'utilité de leur réalisation pour les citoyens ciblés par ces projets. Elles ne constituent pas, en ce sens, des outils efficaces d'aide à la décision. 


\section{DISCUSSION DES RÉSULTATS DE LA RECHERCHE}

Les résultats font ressortir la nature parcellaire des documents. Sauf exception, ils constituent des « catalogues » de projets potentiels supportés par des études de faisabilité techniques sans études complètes de faisabilité.

La recherche a consisté à comparer des documents de planification de projets récréotouristiques en milieu naturel au modèle théorique d'étude de faisabilité de projet. Les résultats font ressortir la nature parcellaire des documents. Sauf exception, ils constituent des «catalogues » de projets potentiels supportés par des études de faisabilité techniques sans études complètes de faisabilité

L'absence de faisabilité de marché empêche, entre autres, de réaliser une faisabilité technique adaptée aux usagers visés par le projet. Citoyens ou touristes? Le projet n'est pas le même en fonction de ces clientèles.

\section{LES IMPLICATIONS MANAGÉRIALES}

Il est possible de synthétiser par deux questions les difficultés engendrées par l'absence d'études complètes de faisabilité : Des projets pour qui? Des projets par qui? Ces deux questions, comme leitmotiv, surgissent obstinément de la lecture des documents de planification de projets. Une multitude de documents, une multitude de projets, mais que des études techniques.

\subsection{Des projets pour qui?}

Qui bénéficiera du projet? Le démarrage de projets récréotouristiques se heurte à cet impératif. Le premier est celui du «pour qui?» L'identification et le choix de projets communs à une collectivité imposent de rendre explicite ce qui est implicite. Dans les plans de développement intégré, est implicite, immatériel et innommé cet individu utilisateur ou cette collectivité bénéficiaire. La réponse à la question «pour qui ces projets?»doit être rendue explicite.

Particulièrement dans le contexte de projets récréotouristiques énoncés par des organismes
Les réponses d'une étude de faisabilité technique rendent hasardeuses les solutions mises de l'avant sans faisabilité complète. Par exemple, le montage financier d'un projet dépend du type d'individu visé par un projet: un citoyen déjà payeur par ses taxes ou un touriste à qui l'on demandera une contribution monétaire. Le manque d'étude de faisabilité de ressources humaines camoufle les besoins de compétence et de leadership des projets, notamment ceux qui requièrent des compétences pointues.

- À la question de départ, à savoir si l'utilisation d'un modèle théorique de faisabilité de projet peut enrichir la pratique de la planification de projets récréotouristiques en milieu naturel, la réponse qu'apporte cette recherche est un oui sans équivoque. L'absence quasi systématique d'études de marché, de faisabilité financière et de ressources humaines conserve aux projets leur aspect « potentiel » et n'aide pas à leur réalisation. Elle rend difficile leur évaluation.

publics, les bénéficiaires doivent être clairement identifiés. Toutes les caractéristiques du projet sont alors conçues en fonction des attentes de ces groupes. Deux cas de figure se présentent.

\section{Dans les plans de développement intégré, est implicite, immatériel et innommé cet individu utilisateur ou cette collectivité bénéficiaire.}

- Les bénéficiaires sont les citoyens du territoire. L'étude de faisabilité de marché devra montrer le désir de la population concernée de bénéficier du projet et préciser les attentes. L'étude de faisabilité technique décrira le projet sous ses aspects matériels. L'étude de faisabilité financière indiquera qui seront les payeurs. Il est raisonnable de s'attendre à ce que l'essentiel du montage financier provienne des communautés concernées et des gouvernements de tutelle. Ils ont la responsabilité de participer au bien-être de leurs citoyens qui sont, de plus, les payeurs ultimes par leurs taxes et leurs impôts. Même si nous 
n'avons trouvé aucune étude de faisabilité de ressources humaines, sa réalisation s'impose $\mathrm{du}$ fait de la nécessité d'identifier les individus et les structures territoriales qui assumeront la direction et le leadership desdits projets.

- Les bénéficiaires sont les touristes et, en corollaire, les gens d'affaires. L'étude de faisabilité de marché devra décrire les désirs et les intentions de comportement de la clientèle. L'étude de faisabilité technique décrira les choix faits pour que les aspects matériels du projet répondent aux attentes de la clientèle et aux impératifs financiers du projet. L'étude de faisabilité financière indiquera qui sont les promoteurs et comment ils comptent financer le projet. Elle contiendra aussi les prévisions financières de rendement sur l'investissement. Il sera raisonnable de s'attendre à ce que ce soient les bénéficiaires financiers des projets qui en assument les risques. L'étude de faisabilité de ressources humaines viendra décrire la gouvernance des projets et les besoins en main-d'œuvre.

Le projet de développement d'un sentier pédestre, par exemple, doit être clairement identifié «pour la collectivité locale» ou « comme attraction touristique ». Les usagers locaux accepteront de partager le sentier, l'hiver, entre quelques skieurs, quelques raquetteurs et quelques marcheurs. Ce sera une tout autre histoire de skier dans un «sentier local » où des centaines de touristes l'auront glacé durant l'avant-midi. À l'inverse, l'industrie locale aura intérêt à offrir des services connexes aux usagers de sentiers pédestres s'ils attirent suffisamment de clients.

Ce qui est à tous n'est à personne. Depuis que la recherche en marketing se penche sur cette question, elle arrive toujours à la même réponse : ce qui «devrait plaire à tous ne plaît à personne».

Dans ce paradigme de l'acteur politique mobilisateur, le locus de contrôle, c'est-àdire la structure territoriale qui devrait exercer le pouvoir de décision sur les projets, demeure à définir.

\subsection{Par qui?}

Les organismes publics peuvent assumer euxmêmes divers projets de développement, particulièrement s'ils sont sur des terres publiques et destinées à la population locale ou régionale. Cependant, il apparaît qu'à moins d'un changement «métamorphique » des politiques publiques québécoises, les organismes de développement ne peuvent être les opérateurs de projets à but lucratif ni être les initiateurs de leur mise en œuvre, et ce, même s'ils l'ont été dans le passé avec bénéfice pour les communautés régionales. On s'attend maintenant à ce que l'acteur politique agisse comme mobilisateur et s'assure que le projet suit les orientations.

Dans ce paradigme de l'acteur politique mobilisateur, le locus de contrôle, c'est-à-dire la structure territoriale qui devrait exercer le pouvoir de décision sur les projets, demeure à définir. Notre étude ne permet pas de la suggérer. Toutefois, la problématique que nous avons identifiée de la parcellisation de l'offre récréotouristique, de l'absence de liens entre les sites, les activités et les acteurs économiques implique que la réponse à la question du locus du contrôle devra permettre cette coordination.

\subsection{Les limites de la recherche}

Cette recherche a porté sur les plans de développement d'une région précise du Québec, l'Outaouais rural. Un examen sommaire de documents provenant d'autres régions rurales $d u$ Québec amène à penser que les conclusions sont généralisables à la plupart des planifications des régions rurales. Les grands centres urbains sont structurés différemment. Les conclusions de cette recherche peuvent ne pas s'appliquer aux documents de planification de ces centres urbains.

\section{BIBLIOGRAPHIE}

${ }^{1}$ Bourgault, Normand (2013). Analyse et synthèse des voies de développement en récréotourisme de l'Outaouais rural - Portrait et enjeux, Université du Québec en Outaouais, avril, 108 pages.

${ }^{2}$ Bourgault, Normand (2006). MGP 7150 : Faisabilité de projet - Notes de cours. Université du Québec en Abitibi-Témiscamingue, 339 pages. 